ARTICLE

Received 31 Aug 2013 | Accepted 8 Jan 2014 | Published 3 Feb $2014 \quad$ DOl: 10.1038/ncomms4221

\title{
Strong Schottky barrier reduction at Au-catalyst/ GaAs-nanowire interfaces by electric dipole formation and Fermi-level unpinning
}

Dmitry B. Suyatin', Vishal Jain 1,2, Valery A. Nebol'sin³, Johanna Trägårdh', Maria E. Messing ${ }^{1}$, Jakob B. Wagner ${ }^{4}$, Olof Persson $^{5}$, Rainer Timm ${ }^{5}$, Anders Mikkelsen ${ }^{5}$, Ivan Maximov ${ }^{1}$, Lars Samuelson ${ }^{1}$ \& Håkan Pettersson ${ }^{1,2}$

Nanoscale contacts between metals and semiconductors are critical for further downscaling of electronic and optoelectronic devices. However, realizing nanocontacts poses significant challenges since conventional approaches to achieve ohmic contacts through Schottky barrier suppression are often inadequate. Here we report the realization and characterization of low $n$-type Schottky barriers ( $\sim 0.35 \mathrm{eV}$ ) formed at epitaxial contacts between Au-In alloy catalytic particles and GaAs-nanowires. In comparison to previous studies, our detailed characterization, employing selective electrical contacts defined by high-precision electron beam lithography, reveals the barrier to occur directly and solely at the abrupt interface between the catalyst and nanowire. We attribute this lowest-to-date-reported Schottky barrier to a reduced density of pinning states $\left(\sim 10^{17} \mathrm{~m}^{-2}\right)$ and the formation of an electric dipole layer at the epitaxial contacts. The insight into the physical mechanisms behind the observed lowenergy Schottky barrier may guide future efforts to engineer abrupt nanoscale electrical contacts with tailored electrical properties.

\footnotetext{
${ }^{1}$ Division of Solid State Physics and The Nanometer Structure Consortium (nmC@LU), Lund University, Box 118, SE-221 00 Lund, Sweden. 2 Department of Mathematics, Physics and Electrical Engineering, Halmstad University, Box 823, SE-301 18 Halmstad, Sweden. ${ }^{3}$ Voronezh State Technical University, Moskovskii pr. 14, RU-394026 Voronezh, Russia. ${ }^{4}$ Division of Polymer and Materials Chemistry, Lund University, Box 124, SE-221 00 Lund, Sweden. ${ }^{5}$ Division of Synchrotron Radiation Research and The Nanometer Structure Consortium, Lund University, Box 118, SE-221 00 Lund, Sweden. Correspondence and requests for materials should be addressed to H.P. (email: hakan.pettersson@hh.se).
} 
E lectrical contacts between metals and semiconductors are fundamentally important for semiconductor devices ${ }^{1-3}$. Shrinking their dimensions poses significant challenges because the properties of metal/semiconductor contacts at the nanoscale may differ significantly from those of their macroscopic counterparts, and the same approaches to control these properties are not always suitable at the nanoscale ${ }^{4-9}$. In particular, standard approaches to realize ohmic contacts by suppressing Schottky barriers through heavy doping and/or varying semiconductor chemical composition in the contact region by, for example, an alloy formation ${ }^{10}$ are inadequate at the nanoscale.

One promising type of nanoscale electrical contact that may help alleviate this problem is the epitaxially formed contact between a semiconductor nanowire (NW) and the corresponding metal-catalyst nanoparticle used to grow it. Such contacts have been reported to exhibit a reduced Schottky barrier influence on the contact properties ${ }^{11-13}$. Possible mechanisms behind this interesting behaviour were attributed to an increased role of contact periphery effects compared with microscale contacts ${ }^{11}$, midgap states induced at the contact interface ${ }^{12}$ and Fermi-level unpinning at the contact interface ${ }^{13}$. However, the origin of the observed reduction of the Schottky barrier influence is still far from fully understood.

For the specific case of nanocontacts formed at Au-catalyst/ GaAs-NW interfaces-such systems are particularly interesting because of their technological importance ${ }^{14}$-a reduction of the $n$-type Schottky barrier height from the normally observed value of 0.9 to $0.52 \mathrm{eV}$ was reported ${ }^{13}$. In that case, the Schottky barrier lowering was attributed to an alleviated Fermi-level pinning. However, because of limited precision in contacting the Aucatalyst in the experiment there was also an overlap between the thin film electrode and the NW segment below the Au catalyst, which makes the interpretation of the results more challenging as this overlap may, for example, change the field distribution in the semiconductor region. Furthermore, it was unclear whether the nanocontact properties could be further improved beyond what was previously reported ${ }^{13,14}$. It therefore remains unclear whether and how low-energy Schottky barriers can be realized at metalcatalyst/NW interfaces.

Here we demonstrate and characterize by high-precision selective contacts defined by electron beam lithography (EBL) a surprisingly low, $\sim 0.35 \mathrm{eV}, n$-type Schottky barrier that is formed directly and solely at the abrupt epitaxial interface between an Au-In alloy catalytic tip (Au catalyst later in the text) and a GaAs NW. The strong reduction of the Schottky barrier height (considerably lower barrier than the typically observed $0.9 \mathrm{eV}$ for ordinary $\mathrm{Au} / \mathrm{GaAs}$ Schottky contacts and the lowest barrier observed at $\mathrm{Au} / \mathrm{GaAs}$ interfaces to date) occurs because of a combination of reduced density of pinning states and the formation of an electric dipole layer at the Au-catalyst/GaAsNW interface owing to the termination of the GaAs NW by As atoms. Insight about these barrier-lowering mechanisms may enable Schottky barrier height control and low-ohmic abrupt electrical contacts to be formed at metal-semiconductornanostructure interfaces without the need for annealing or contact doping, otherwise limiting factors in rapid downscaling of nanodevices.

\section{Results}

Experimental design. To obtain a comprehensive understanding of the mechanisms behind the Schottky barrier formation at metal-catalyst/NW interfaces, we considered two different types of NWs, as well as two independent characterization methods. The A series comprised Au-catalyst/GaAs/InGaAs/InAs-NW heterostructures with $90 \mathrm{~nm}$ diameter, grown by chemical beam epitaxy (CBE) on InAs (111)B substrates. The Au catalysts were alloyed with In during the NW growth. These NWs were probed with EBL-defined contacts and characterized by current-voltage $(I-V)$ measurements at different temperatures and under illumination. More details on the NW growth can be found in Methods and elsewhere ${ }^{15}$.

The B series comprised Au-catalyst/GaAs-NWs with $95 \mathrm{~nm}$ diameter, grown by metal organic vapour phase epitaxy (MOVPE) on GaAs (111)B n-type substrates. The Au catalysts were alloyed with In before the NW growth. These NWs were used for complementary $I-V$ measurements at room temperature by direct contacting of the Au-catalyst particles with the tip of a scanning tunnelling microscope (STM) to verify the observed Schottky barrier height. More details on the NW growth can be found in Methods and elsewhere ${ }^{16}$. Both types of NWs (series A and B) grew perpendicular from the substrate indicating that the epitaxial contact was formed at the Au-catalyst/GaAs (111)B NW interface.

The chosen NW design in our study was motivated by the following: first, the selective electrical contacting to the $\mathrm{Au}$ catalyst was realized with a high-precision EBL alignment scheme, which ensured that electron transport occurred solely through the epitaxial Au-catalyst/GaAs-NW interface. The EBLdefined contacts also enabled detailed characterization, including $I-V$ measurements at different temperatures and under light excitation. Second, the heterostructure NW design implemented for the NWs in series $\mathbf{A}$ allowed the formation of electrical contacts to the Au-catalyst/GaAs-NW interface from the NW side through an ohmic contact to the InAs NW segment ${ }^{17}$ via a graded InAs/InGaAs/GaAs heterojunction. In this way, deleterious effects from parasitic electrical contacts were eliminated. Third, the NW diameter used in our experiments allows a direct comparison with previous studies ${ }^{11,13}$, while at the same time ensuring that quantum mechanical confinement effects do not significantly influence the observed phenomena. Fourth, the experimental design ensured that a wide enough potential barrier was formed at the Au-catalyst/GaAs-NW interface to avoid any tunneling effects. Finally, the alloying of the Au catalyst with In in our experiments was done because In/GaAs-alloyed contacts are known to exhibit a low Schottky barrier height of merely $0.03 \mathrm{eV}$ (ref. 18).

Series A NW characterization. To elucidate the structural and elemental composition of the heterostructure NWs from series A, we performed a transmission electron microscopy (TEM) and X-ray energy-dispersive spectroscopy (XEDS) analysis (Fig. 1); more details on the NW inspection with TEM can be found in Methods and Supplementary Table 1. The TEM image reveals that an abrupt contact is formed between the NW and the metal particle (inset of Fig. 1 and Supplementary Fig. 1). The XEDS analysis shows that the length of the pure GaAs segment right under the Au catalytic particle was $\sim 25 \mathrm{~nm}$, while the graded InGaAs segment was $\sim 75 \mathrm{~nm}$ with a smooth transition from pure GaAs to pure InAs. Note that the line scan presented in Fig. 1 shows an approximate element distribution in the NW as the XEDS count normalization needs to be done differently in different regions.

The XEDS point measurements revealed a $31 \pm 1$ at. $\%$ In content in the catalytic particle, whereas the $\mathrm{Ga}$ and As contents were found to be below the detection limit of 1-2 at.\% (Supplementary Table 1). No GaAs shell was observed around the InAs segment. These results demonstrate that the Au catalysts in our series A NWs were alloyed with In only. Furthermore, there was no Au-alloying of the GaAs and thus, an abrupt contact was formed between the NW and the metal particle. 


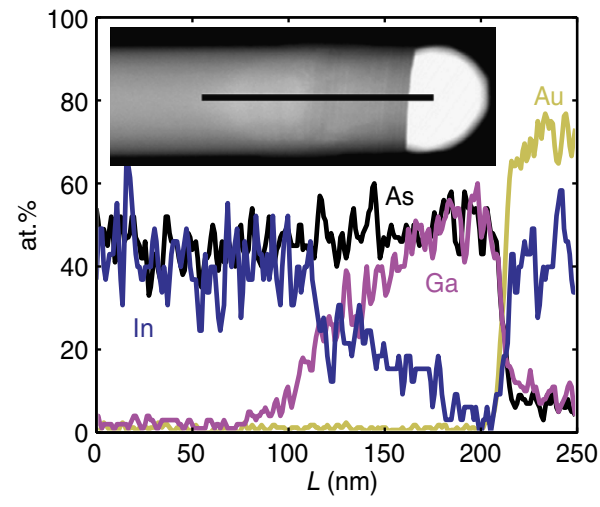

Figure 1 | XEDS line scan for a series A heterostructure NW. The XEDS scan was done along the line indicated in the TEM micrograph (inset). Note that the line scan shows an approximate element distribution in the NW as the XEDS count normalization needs to be done differently in different regions. XEDS point measurements revealed a $31 \pm 1$ at. $\%$ In content in the catalytic particle, whereas the $\mathrm{Ga}$ and As contents were found to be below the detection limit (1-2 at.\%; Supplementary Table 1).

To enable detailed electrical characterization of the catalyst/ NW interfaces, we formed electric contacts selectively to the catalytic particle using EBL (inset of Fig. 2). To the best of our knowledge, this is the first time a NW catalyst particle has been selectively contacted by EBL. It was made possible owing to a high-precision alignment before the EBL exposure, which allowed a control of the electric contact position with $10-20 \mathrm{~nm}$ precision (more details on electrical contact formation can be found in Methods and elsewhere ${ }^{17}$ ).

Electrical measurements on the series A NWs revealed highly asymmetric, rectifying $I-V$ characteristics at different temperatures indicating electron transport governed by a thermionic emission (TE) over an $n$-type potential barrier (Fig. 2, inset shows a schematic picture of the electrical circuit used in the measurements). The two possible positions along the NW at which a potential barrier may be formed are at the Au-catalyst/ GaAs-NW interface and in the GaAs/InGaAs-NW segment. As we will show in the following analysis, the relevant potential barrier determining the electron transport at large reverse bias is formed at the Au-catalyst/GaAs-NW interface.

A model based on TE of electrons is used to evaluate the properties of this potential barrier. According to TE theory, the $I-V$ characteristics for $V_{\text {app }}<-3 k T / q$ are described by:

$$
\ln \left(-\frac{I}{T^{2}}\right)=\ln \left(A A^{*}\right)-\frac{q \phi_{\mathrm{E}}\left(V_{\text {app }}\right)}{k T}
$$

where $V_{\text {app }}$ is the applied voltage, $I$ is the current through the device, $A$ is the effective contact area between the catalytic particle and the NW, $A^{*}$ is the Richardson constant and $q \phi_{\mathrm{E}}\left(V_{\mathrm{app}}\right)$ is the effective voltage-dependent energy barrier height (for details see Methods and elsewhere ${ }^{1,19}$ ). Arrhenius plots (Fig. 3b) were extracted from temperature-dependent $I-V$ measurements (Fig. 3a) and analysed using equation (1). Figure $3 c$ shows the extracted barrier height $q \phi_{\mathrm{E}}\left(V_{\mathrm{app}}\right)$ for different bias $V_{\mathrm{app}}$. The results reveal that the barrier height for small applied bias $\left(V_{\text {app }}>-0.1 \mathrm{~V}\right.$, negative bias applied to the Au particle with respect to the grounded InAs segment of the wire) is $\sim 0.5 \mathrm{eV}$, while for large bias $\left(V_{\text {app }}<-0.4 \mathrm{~V}\right)$ the barrier height saturates at $0.35 \pm 0.03 \mathrm{eV}$. Since equation (1) is valid only for $V_{\text {app }}<-3 k T / q$ $(\sim 0.08 \mathrm{~V})$, the extracted values close to zero bias are not

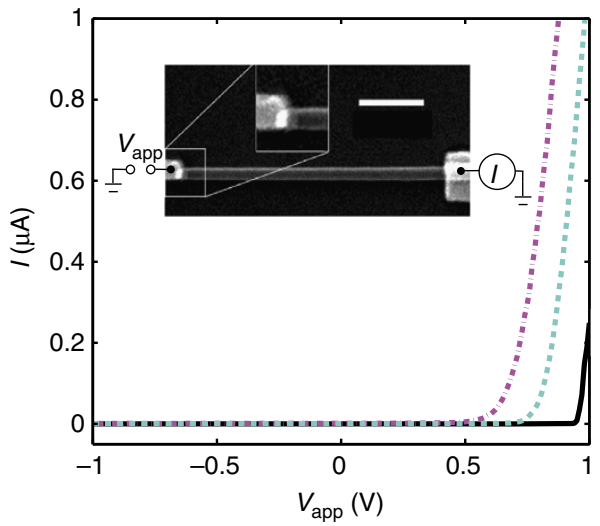

Figure 2 | I-V characteristics for a heterostructure NW device from series A. I-V characteristics recorded at $4.2 \mathrm{~K}$ (black solid line), $300 \mathrm{~K}$ (turquoise dashed line) and $410 \mathrm{~K}$ (magenta dash-dotted line). The inset shows a scanning electron microscope (SEM) image of the measured device and the schematic electrical circuit set-up for the measurements. Scale bar, $500 \mathrm{~nm}$. Zoomed region shows the selective electrical contact formed to the catalytic particle.
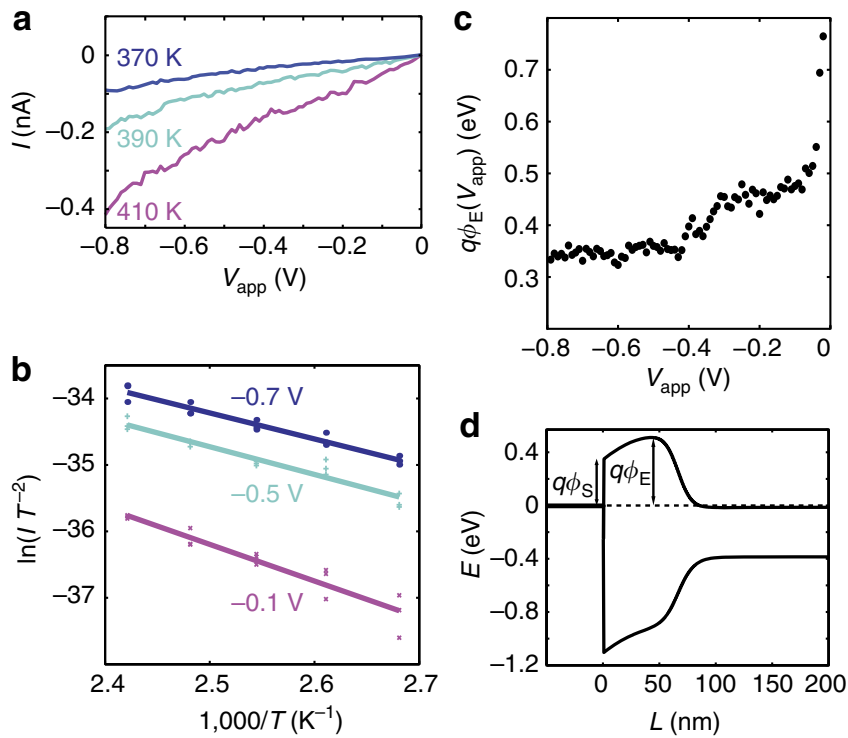

Figure 3 | The Schottky barrier height extraction for a heterostructure NW device from series A. (a) $I-V$ characteristics at different temperatures in the reverse bias region. (b) Arrhenius plots extracted from I-V curves measured in the temperature range $370-410 \mathrm{~K}$. (c) The extracted effective voltage-dependent energy barrier height $q \phi_{\mathrm{E}}\left(V_{\mathrm{app}}\right)$ plotted versus applied bias $V_{\text {app }}$. Negative bias corresponds to a reverse-biased Schottky contact; $c f$. the inset in Fig. 2. This analysis is only valid for $V_{\text {app }}<-3 k T / q$, which explains the large scattering observed close to zero bias. (d) Band diagram reconstruction of the studied NW heterostructure at thermal equilibrium. Here $q \phi_{\mathrm{E}}$ indicates the energy barrier height at zero bias.

accurately described by equation (1) and therefore the scattered points in this region are not meaningful.

To interpret these results we reconstructed the band diagram shown in Fig. 3d. As input for this reconstruction, we used parameters known for this type of NWs and the barrier heights extracted from our experiments at $V_{\text {app }}>-0.1 \mathrm{~V}$ and $V_{\text {app }}<-0.4 \mathrm{~V}$ (for details see Methods and elsewhere ${ }^{20}$ ). The 
results show that the entire GaAs/InGaAs segment is depleted from free carriers. As seen in the diagram, the potential barrier that forms at thermal equilibrium exceeds the $n$-type Schottky barrier height, $q \phi_{\mathrm{S}}$. The reason for this is that $q \phi_{\mathrm{S}}<q \phi_{\mathrm{GaAs}^{-}}$ $q \chi_{\mathrm{GaAs}}$, where $q \phi_{\mathrm{GaAs}}$ is the work function and $q \chi_{\mathrm{GaAs}}$ is the electron affinity of the GaAs NW segment. Therefore, a negative $V_{\mathrm{app}}$ has to be applied to reach conditions where $q \phi_{\mathrm{S}}$ limits electron transport. We interpret the flat region for $V_{\text {app }}<-0.4 \mathrm{~V}$ (Fig. 3c) as the region where the electron transport is determined by the Schottky barrier between the Au catalyst and GaAs NW for which we extract $q \phi_{\mathrm{S}}=0.35 \pm 0.03 \mathrm{eV}$. This value is considerably smaller than $0.9 \mathrm{eV}$ known for abrupt $n$-type bulk Au/GaAs Schottky contacts ${ }^{21,22}$. In fact, to the best of our knowledge, this is the smallest Schottky barrier height ever reported for $\mathrm{Au} / \mathrm{GaAs}$ interfaces. An important comment to this analysis is that the effective contact area $A$ between the Au catalyst and GaAs NW most likely depends on the applied bias ${ }^{23} V_{\text {app }}$ owing to self-gating effects ${ }^{24}$ in combination with a surface depletion $^{23,25}$ due to NW surface oxidation ${ }^{26,27}$. However, such effects will not affect the extracted barrier height, as evident from equation (1) as well as from the saturation of the barrier height observed in Fig. 3c. The band diagram in Fig. $3 \mathrm{~d}$ represents the potential profile along the core of the $\mathrm{Au}$-catalyst/NW that determines the thermionic current ${ }^{3}$. This one-dimensional core profile is realistic also if one considers three-dimensional boundary conditions, for example, a Fermi-level position on the sidewall surface of the NW $<0.7-0.9 \mathrm{eV}$ below the conduction band (values known for oxidized GaAs surfaces) ${ }^{28}$. The soft reverse $I-V$ characteristics observed (Fig. 3a) can also be understood from this model ${ }^{25}$ as a self-gating effect ${ }^{24}$ where the reverse current continues to increase with bias (Fig. 3a), although the effective potential barrier $q \phi_{\mathrm{E}}\left(V_{\mathrm{app}}\right)$ saturates for $V_{\text {app }}<-0.4 \mathrm{~V}$ (Fig. 3d). The increasing reverse current results from an increase of the effective cross-section of the saddle point, which is decoupled from the Schottky barrier height in our analysis (for details see Methods).

It is also important to note that our interpretation of the experimental results is based on the assumption that TE is the dominant electron transport mechanism. If another current component, comparable to the TE current in magnitude, existed then the extracted barrier height could be misestimated. Indeed, the following current components besides TE current can be observed in a metal-semiconductor junction ${ }^{1}$ : (i) quantum mechanical tunnelling through the barrier and (ii) electron-hole generation-recombination. The $\mathrm{NW}$ design in our case was chosen in such a way that TE dominates. The InAs segment is unintentionally degenerately $n$-type doped (electron concentration $\left.\sim 10^{17} \mathrm{~cm}^{-3}\right)^{29}$, while the GaAs segment is nondegenerately $p$-type doped (with the same doping concentration $)^{30}$. The length of the GaAs segment ensures a complete depletion of free carriers and that the potential barrier formed suppresses any tunnelling effects. Moreover, the GaAs segment creates an efficient potential barrier for both electrons and holes and the generation-recombination currents due to deep levels in $\mathrm{Au} / \mathrm{GaAs}$ Schottky diodes are typically negligible ${ }^{3 \mathrm{f}}$. Indeed, at $4.2 \mathrm{~K}$ we did not observe any current $\left(<10^{-12} \mathrm{~A}\right)$ for the voltage range used in our analysis, indicating that there is no tunnelling current or other currents at low temperature. However, temperature is known to have a profound effect on thermally activated tunnelling (thermionic field emission; TFE) and generation-recombination currents ${ }^{32}$. Linear characteristics in the Arrhenius plots in Fig. 3b indicate that TE dominates in our case since TFE and generation-recombination currents exhibit different temperature dependences ${ }^{10}$ and any significant contribution to the TE current would result in nonlinear characteristics in the Arrhenius plots.
Series B NW characterization. To verify that the observed Schottky barrier height is not specific only to NWs from series A, and as a validation of the Schottky barrier height analysis, we carried out complementary $I-V$ measurements at $300 \mathrm{~K}$ on NWs from the $\mathbf{B}$ series using STM under ultra-high vacuum conditions (see Methods and elsewhere ${ }^{33}$ for details).

Figure 4 shows typical $I-V$ data recorded with the STM on nominally undoped NWs with $95 \mathrm{~nm}$ diameter and $\sim 500 \mathrm{~nm}$ length. The highly asymmetric, rectifying $I-V$ curve (Fig. 4) indicates that the electron transport is mainly determined by an $n$-type potential barrier. The red line in the inset shows a linear least-square fit of the semi-log $I$ versus $V_{\text {app }}$ for $V_{\text {app }}>>k T / q$. Extrapolating this line to $V_{\text {app }}=0$ gives the saturation current $I_{s}$, which was used to calculate $q \phi_{\mathrm{S}}$ according to:

$$
q \phi_{\mathrm{S}}=k T \ln \left(\frac{A A^{*} T^{2}}{I_{\mathrm{S}}}\right)
$$

From this analysis we obtained $q \phi_{\mathrm{S}}=0.32 \pm 0.04 \mathrm{eV}$ for NWs from series $\mathbf{B}$, which is in good agreement with the $q \phi_{\mathrm{S}}$ deduced for the NWs in the A series discussed above (see Methods and elsewhere ${ }^{1,19}$ for more details).

It is also important to note that in this case we observe a large ideality factor $n=2.6 \pm 0.2$. Traditionally, the ideality factor was used to determine the deviation of electron transport from TE. However, as recognized already in the $1990 \mathrm{~s}^{3}$, the ideality factor cannot be used for the determination of transport mechanisms, especially for NW geometries ${ }^{25}$. Our experimental design ensured that the effect of TE dominates over TFE since $k T>>E_{00}$, where $E_{00}$ is a property of the semiconductor indicating how efficient tunnelling is in the semiconductor ${ }^{3}$. For the studied NWs, $E_{00}<7 \mathrm{meV}$ (since the NW geometry increases the depletion region ${ }^{11,26}$ ) while the measurements were done at room temperature $(k T=26 \mathrm{meV})$. Furthermore, a theoretical value of the expected low-bias differential conductance for $\mathrm{TE}^{11}$ given by $\mathrm{d} J / \mathrm{d} V=\left(q A^{*} T^{2} / \mathrm{kT}\right) \exp \left(-q \phi_{\mathrm{S}} / k T\right)$ at $300 \mathrm{~K}$ with $A^{*}=7 \mathrm{~A} \mathrm{~cm}^{-2} \mathrm{~K}^{-2}$ and $q \phi_{\mathrm{S}}=0.35 \mathrm{eV}$ yields $32 \mathrm{~A} \mathrm{~V}^{-1} \mathrm{~cm}^{-2}$ This value is in good agreement with the experimental value of $30 \mathrm{~A} \mathrm{~V}^{-1} \mathrm{~cm}^{-2}$ deduced from our STM measurements. We therefore attribute the observed large ideality factor to surface depletion $^{23,25}$ due to NW surface oxidation ${ }^{26,27}$, an effect that we discussed above for NWs from series $\mathbf{A}$.

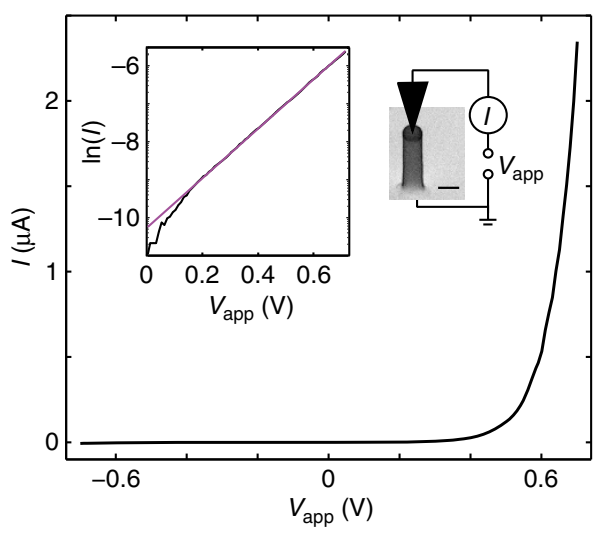

Figure 4 | I-V characteristics for a series B NW. STM-based measurements of $I-V$ characteristics for a nominally undoped GaAs NW at $300 \mathrm{~K}$. The left inset shows a semi-log plot with a linear fit in the forward bias region (magenta line). The right inset shows an SEM micrograph of a typical NW together with the schematic electrical circuit used for the measurements. Scale bar, $100 \mathrm{~nm}$. 
Mechanisms responsible for the low Schottky barrier. To elucidate the physics behind this surprisingly low Schottky barrier, we will discuss several mechanisms that could be responsible for this result. First, the low barrier height observed in our experiments are not caused by annealing because the interface between the epitaxial Au-catalyst/GaAs-NW is abrupt according to TEM/ XEDS, in contrast to the non-uniform Au diffusion into GaAs previously observed after annealing at $350{ }^{\circ} \mathrm{C}$ (ref. 14). This annealing resulted in a high dislocation density in the GaAs and the lowest-to-date barrier height for macroscopic $\mathrm{Au} / \mathrm{GaAs}$ $n$-type Schottky contacts of $\sim 0.5 \mathrm{eV}$.

Second, a reduced Fermi-level pinning was previously suggested as a possible cause for lowering Schottky barriers at metal-catalyst/NW interfaces ${ }^{13}$. We suggest this effect to play a major role in our case since our observations do not fit the empirically known behaviour for abrupt metal contacts to $n$-type GaAs where the Schottky barrier depends on the metal work function $q \phi_{\mathrm{M}}$ according to $q \phi_{\mathrm{S}}=0.49+0.074 q \phi_{\mathrm{M}}$ (refs 34,35). In case of pure $\mathrm{Au}\left(q \phi_{\mathrm{M}}=5.31 \mathrm{eV}\right.$ (ref. 36)) in contact with GaAs, the corresponding Schottky barrier is expected to amount to $q \phi_{\mathrm{S}(\mathrm{Au})}=0.88 \mathrm{eV}$. Possible effects of In mixing in the Au catalyst also cannot explain the lowering since $q \phi_{\mathrm{In}}=4.12 \mathrm{eV}$ (ref. 36), which corresponds to $q \phi_{\mathrm{S}(\mathrm{In})}=0.79 \mathrm{eV}$ for a pure In contact to GaAs. A reduced density of interface pinning states was previously suggested to be responsible also for interface Fermilevel unpinning in GaAs grown at low temperature ${ }^{37}$.

However, our observations cannot be explained solely by a reduced density of pinning states because a comparison with an ideal Schottky model reveals higher barrier values than we observe. According to the ideal Schottky model ${ }^{1}$, which does not consider Fermi-level pinning, the $n$-type Schottky barrier height depends on the contact metal work function as $q \phi_{\mathrm{S}}=q \phi_{\mathrm{M}}-q \chi$, where $q \chi$ is the semiconductor affinity, $q \chi_{\mathrm{GaAs}}=4.07 \mathrm{eV}$ (ref. 38). The work function of solid solution alloys can be described by a simple mixture rule of the surface composition: $q \phi_{\mathrm{AuIn}}=q x_{\mathrm{Au}} \phi_{\mathrm{Au}}+q x_{\mathrm{In}} \phi_{\mathrm{In}}$, where $x_{i}$ is the atomic fraction of element $i$ at the surface ${ }^{39}$. This expression yields $q \phi_{\text {AuIn }}=4.96 \mathrm{eV}$ for a 30 at.\% In content in the Au catalyst, which corresponds to $q \phi_{\mathrm{S}(\mathrm{AuIn})}=0.89 \mathrm{eV}$, a considerably higher value than we observe in our experiments.

We explain the barrier lowering by a reduced density of pinning states combined with a formation of an electric dipole layer. The dipole layer is formed because of the termination of the GaAs NW by As atoms at the contact interface with the $\mathrm{Au}$ catalyst. The Au-catalyst/GaAs-NW interface studied in this work had a (111)B structure as the NWs grew vertically on (111)B substrates. This (111)B GaAs plane has only As atoms at the interface. Since As atoms in contact with an Au catalyst have higher electronegativity than $\mathrm{Ga}$ atoms, an interfacial electric dipole layer is formed with a dipole moment $\boldsymbol{\mu}$ pointing towards the semiconductor NW and an associated electric field $\mathbf{E}$ directed towards the Au catalyst (Fig. 5). This electric field leads to an effective reduction of the Schottky barrier. The ideal Schottky model (Fig. 5a) predicts the Schottky barrier for the In-alloyed Au-catalyst $/ \mathrm{GaAs}-\mathrm{NW}$ interface of $q \phi_{\mathrm{S}(\mathrm{AuIn})}=0.89 \mathrm{eV}$ discussed in the previous paragraph. The electric field associated with the dipole layer leads to a step in the potential energy at the interface (Fig. 5b), which shifts the conduction band edge of the GaAs NW below the Fermi level in the Au-catalyst particle by an amount $q \Delta=0.33 \mathrm{eV}$ (see Methods for details). If we now assume that pinning states at the interface account for the potential energy $q d \phi_{\text {States }}=q \phi_{\mathrm{S}}+q \Delta=0.68 \mathrm{eV}$, we can estimate a density of surface states at the Au-catalyst/GaAs interface of $\sim 10^{17} \mathrm{~m}^{-2}$ (see Methods for details). Remarkably, as it was previously shown $^{40}$, this density of surface states implies that the Fermi level is no longer pinned at the interface, and both the surface states
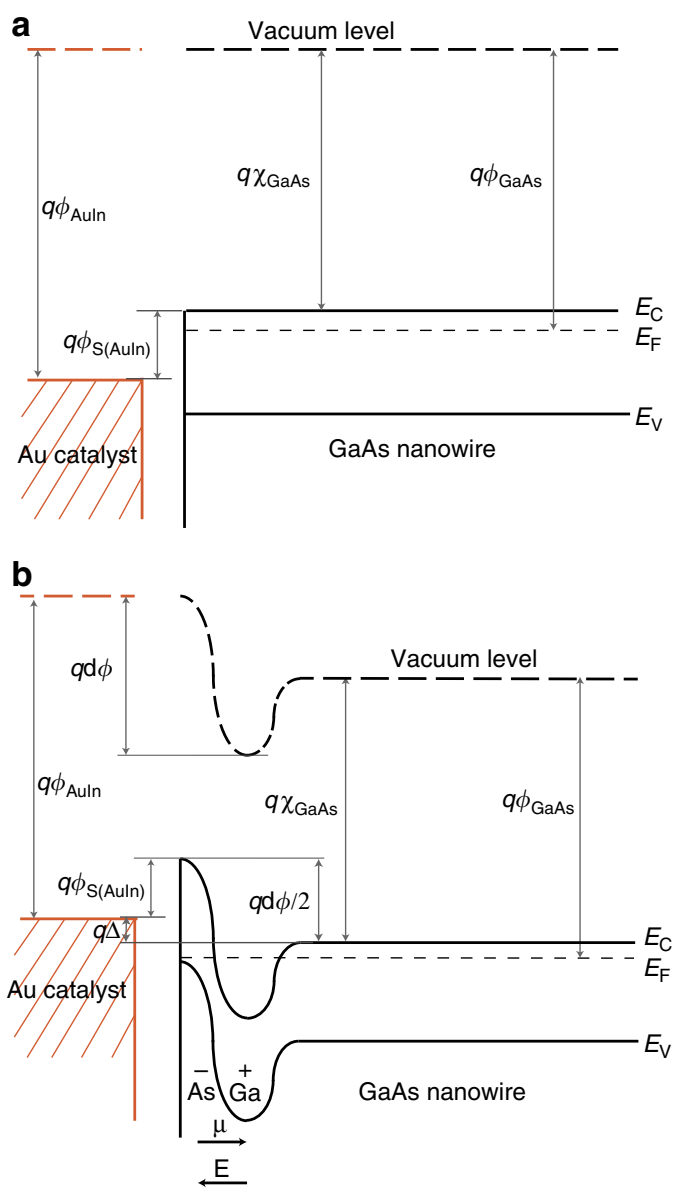

Figure 5 | Energy band alignment of an isolated Au catalyst and n-type GaAs (111)B NW. (a) Ideal Schottky model. (b) Ideal Schottky model with included electric surface dipole layer formed because of As termination of the GaAs NW.

and the work function difference will affect the Schottky barrier height.

Based on these arguments, we thus conclude that our observation of the drastic decrease in Schottky barrier height in our NW systems is caused by the combined effects of a reduced density of pinning states $\left(\sim 10^{17} \mathrm{~m}^{-2}\right)$ at the epitaxially formed Au-catalyst/GaAs-NW interface and an electric dipole layer formation at the same interface because of the termination of the GaAs NW by As atoms.

It is known that GaAs NWs often have two crystal phases with different band gap alignment and sizes ${ }^{41,42}$. In our samples the presence of two crystal phases is manifested by stacking faults (Supplementary Fig. 1) and it is not possible to conclude whether the Schottky contact in our case was formed with a Zinc blende or with a Wurtzite phase of GaAs. For bulk GaAs, the Zinc blende phase is the only phase possible and thus essentially all Schottky barrier height studies to date (except perhaps a recent report where the phase influence is not discussed ${ }^{13}$ ) were done for this crystal phase. However, as recently shown by different groups $^{41,42}$, the difference in band gap alignment and band gap size is below $0.1 \mathrm{eV}$, which is much smaller than the Schottky barrier reduction observed in our case and comparable to the measurement errors. To the best of our knowledge, the effect of different crystal phases on interface properties and the Schottky barrier height has not been studied so far. However, according to the present understanding of the Schottky barrier height 
dependence on material properties ${ }^{3}$, the crystal phase per se does not affect the Schottky barrier height. Instead, the presence of different defects and the specific interface atomic structure determines the barrier height. In our case, the interface between the catalytic particle and semiconductor NW is terminated by As atoms for both (111)B Zinc blende phase and (000-1) Wurtzite phase interfaces, and thus we do not expect any substantial influence of the crystal phase on the Schottky barrier height observed.

The epitaxial nature of the Au-catalyst/GaAs-NW interface and its small area are likely responsible for the reduced pinning as chemical bonds form naturally in this epitaxial abrupt junction during the NW growth, which reduces the density of surface states. The small junction area likely also facilitates a relaxation of interfacial defects due to the close proximity of the NW surface ${ }^{43}$. The specific electrostatics for nanoscale contacts is also known to provide weaker impact of metal-induced surface states on the Fermi-level pinning 8 .

The reported electric dipole layer contribution to the Schottky barrier height has implications beyond the NW systems studied here. It can also account for the difference in barrier height observed for bulk Schottky contacts formed at semiconductor interfaces with different crystallographic planes. For example, it has been observed for $n$-type Au/GaAs Schottky contacts that the Schottky barrier is $0.06 \mathrm{eV}$ higher for contacts interfacing with (111)A planes compared with those interfacing with (111)B planes $^{44}$. The observed difference in barrier height is likely to be caused by the electric dipole layers at the contact interface as it corresponds to a realistic density of surface states ${ }^{40}$ of $10^{18}$ $10^{19} \mathrm{~m}^{-2} \gg 10^{17} \mathrm{~m}^{-2}$. The proposed Schottky barrier-lowering mechanism may also be relevant for contacts formed at abrupt interfaces with thin films with a reduced density of interfacial pinning states, for example, (111)B GaAs grown at low temperature ${ }^{37}$. To the best of our knowledge, this electric dipole layer effect has not been considered before when describing reduced Schottky barrier heights experimentally observed, although similar effects were previously theoretically predicted ${ }^{3,45}$. Thus, this finding may be of fundamental importance and can potentially enable Schottky barrier height management by controlling the density of pinning states and by designing the surface dipole layer by choosing appropriate crystal orientation and introducing interfacial atoms with appropriate electronegativity. This is important for different applications where optimization of charge transfer processes at semiconductor surfaces is required, for example, in heterogenous photocatalysis and photochemistry ${ }^{46}$. In particular, this finding can be important for realization of low-ohmic abrupt electrical contacts without the need for contact doping or annealing. This would be a major advantage since doping variability is the key challenge for downscaling of electronic devices.

Photocurrent studies. In addition to our investigation of ultralow Schottky barriers formed at Au-catalyst/NW interfaces, the progress in realizing EBL-defined selective contacts to the Aucatalysts opens up the possibility to design and perform new studies combining multiple characterization techniques to probe electron transport at the catalyst/NW epitaxial interface in great detail. In contrast to STM contacting techniques, our approach allows for temperature-dependent and light-assisted $I-V$ measurements, which are typically difficult to perform in STM-based approaches. To demonstrate this versatility, we performed photocurrent measurements on the catalyst/NW epitaxial contacts, which reveal a clear photovoltaic effect and a linear dependence of the short-circuit current on the excitation light power, which is desirable for photodetection applications (Supplementary Fig. 2).
The measurements demonstrate that the selective contacts defined with EBL provide the ability to combine multiple characterization techniques for studying the same catalyst/NW epitaxial interface. This is a crucial advantage as quantifying and controlling material properties, such as carrier concentration and mobility by, for example, spatially resolved Hall measurements or electron beam-induced cathodoluminescence measurements on single $\mathrm{NWs}^{47}$, are essential for providing more insight into electron transport at nanoscale interfaces and engineering nanoscale electrical contacts with desirable electrical properties.

\section{Methods}

Series A NW growth. Series A heterostructure NWs were grown by a custombuilt CBE system from $\mathrm{Au}$ aerosol seed particles with $70 \mathrm{~nm}$ diameter produced by an aerosol method ${ }^{48}$. The particles were deposited on an InAs (111)B substrate with a density of $\sim 0.5$ particles per $\mu \mathrm{m}^{2}$. Before the growth, the samples were annealed at $520^{\circ} \mathrm{C}$ for $20 \mathrm{~min}$ in a tertiarybutylarsine (TBAs) atmosphere in the CBE reactor. The NW growth was carried out at $420^{\circ} \mathrm{C}$ using trimethylindium (TMIn), triethylgallium (TEGa) and TBAs as precursors. The source pressures in the lines before the inlet valves during the growth were $p_{\mathrm{As}}=150 \mathrm{~Pa}$ for TBAs, $p_{\mathrm{In}}=20 \mathrm{~Pa}$ for TMIn and $p_{\mathrm{Ga}}=20 \mathrm{~Pa}$ for TEGa. The NWs were grown about $4 \mu \mathrm{m}$ long, with a resulting diameter of about $90 \mathrm{~nm}$. After growth, precautions were made not to expose the NWs to air for extended periods of time before electrical characterization.

Series B NW growth. Series B NWs were grown by MOVPE (AIX200/4, Aixtron AG) from Au aerosol seed particles with $80 \mathrm{~nm}$ diameter produced by the aerosol method. The particles were deposited on a GaAs (111)B $n$-type substrate (Si doped, $\sim 10^{18} \mathrm{~cm}^{-3}$ doping level) with a density of approximately five particles per $\mu \mathrm{m}^{2}$ Before the growth, the samples were exposed to TMIn for $2 \mathrm{~min}$ at $350{ }^{\circ} \mathrm{C}$ to enrich the seed particles with In to a level comparable to that of the series A NWs. Subsequently, the samples were annealed at $600{ }^{\circ} \mathrm{C}$ for $9 \mathrm{~min}$ in hydrogen $\left(\mathrm{H}_{2}\right)$ and arsine $\left(\mathrm{AsH}_{3}\right)$ atmosphere in the MOVPE reactor. The NW growth at $450{ }^{\circ} \mathrm{C}$ with trimethylgallium (TMGa) and $\mathrm{AsH}_{3}$ precursors (molar fractions $10^{-5}$ and $10^{-4}$ respectively) in a $\mathrm{H}_{2}$ carrier gas flow of $6 \times 10^{-3} \mathrm{~m}^{3} \mathrm{~min}^{-1}$ resulted in vertical GaAs NWs with a length of up to $500 \mathrm{~nm}$ and a diameter of $95 \mathrm{~nm}$. The growth was conducted under low pressure $(10 \mathrm{kPa})$. NW samples with three different doping profiles were grown: fully $n$-type ( $\mathrm{Sn}$ doping with an estimated concentration of $10^{18} \mathrm{~cm}^{-3}$ was introduced during the whole $2 \mathrm{~min}$ NW growth), half-way-doped $n$-type (Sn doping with an estimated concentration of $10^{18} \mathrm{~cm}^{-3}$ was introduced during the first 2 min of the NW growth, followed by a 2 min growth of a nominally undoped NW segment) and nominally undoped. Triethyltin (TESn) was used for the NW doping. After growth, precautions were made not to expose the NWs to air for extended periods of time before electrical characterization.

NW inspection with TEM. A 300-kV TEM (JEOL JEM-3000F) equipped with a field emission gun was used to characterize the A series NWs with a resolution of $0.165 \mathrm{~nm}$. To prepare the samples for TEM analysis, a $\mathrm{Cu}$ grid was rubbed against the substrate surface, which broke off the NWs close to their base.

Formation of electrical contacts to series A NWs. After growth, NWs from the A series were mechanically transferred onto silicon substrates capped with a thermally grown $100 \mathrm{~nm}$ thick silicon dioxide top layer. The substrates had a set of macroscopic metal pads for connection to the external circuitry. The substrates also had predefined reference markers for accurate location of the NWs and alignmen crosses for subsequent EBL alignment. The reference markers and the alignment crosses were made in an EBL process (Raith 150 EBL system, Raith GmbH) by thermal evaporation of $10 \mathrm{~nm}$ Ti and $50 \mathrm{~nm} \mathrm{Au}$ with a subsequent lift-off process.

EBL was used to define selective electrical contacts to the Au-catalyst nanoparticle and to the InAs segment of single NWs. To form selective electrical contacts to Au-catalyst nanoparticles, it was crucial to perform a high-precision alignment of the metal electrodes formed by EBL with respect to the mechanically deposited NWs. For this, the position of individual NWs, relative to the reference markers, was determined by taking images with the SEM of the EBL system. The images were recorded at $20 \mathrm{kV}$ accelerating voltage with a maximum radiation dose limited to $\sim 700 \mu \mathrm{Ccm}^{-2}$, as it was observed that higher doses could damage the NWs. Before recording each image, a field alignment was done using the predefined alignment markers. The specific design (Supplementary Fig. 3) of the reference markers and the alignment crosses, including features with dimensions down to $40 \mathrm{~nm}$, was important for the high-precision alignment process. The sample was coated with a standard e-beam-sensitive resist polymethylmethacrylate (PMMA) after localizing NWs of interest. A $150 \mathrm{~nm}$ thick single layer of PMMA (molecular weight 950,000 Da) was deposited by spinning at 5,000 r.p.m. for $30 \mathrm{~s}$ with a subsequent baking at $180^{\circ} \mathrm{C}$ for $1 \mathrm{~h}$ in an oven. The EBL exposure was done at $20 \mathrm{kV}$ accelerating voltage with a $700 \mu \mathrm{C} \mathrm{cm}^{-2}$ radiation dose. 
The high-precision NW alignment carried out before the electron beam exposure allowed a control of the electric contact position to within $10-20 \mathrm{~nm}$, see inset in Fig. 2. The yield of the selective contacts was found to be up to $80 \%$ and was mostly limited by NW displacement during the e-beam resist spinning. A second EBL step was subsequently carried out to establish the ohmic contact to the InAs segment of the NW and included InAs surface sulphur passivation ${ }^{17}$. The contacts to the Au particle and InAs segment were both fabricated by thermal evaporation of $250 \AA \mathrm{Ni}$ and $900 \AA$ Au with a subsequent lift-off process.

Barrier height analysis for series A NWs. The ideal I-V relation for a metal-semiconductor contact under TE conditions is given by ${ }^{1}$

$$
J=A^{*} T^{2} \exp \left(-q \phi_{\mathrm{E}} / k T\right)\left\{\exp \left(q V_{\text {app }} / k T\right)-1\right\},
$$

where $J$ is the current density per unit area, $A^{*}$ is the Richardson constant, $q \phi_{\mathrm{E}}$ is the effective potential barrier height and $V_{\text {app }}$ is the bias applied, positive for forward bias, that is, positive potential applied to the metal side.

For $V_{\text {app }}<-3 k T / q$ and a bias-dependent effective barrier height $q \phi_{\mathrm{E}}\left(V_{\mathrm{app}}\right)$, equation (3) reduces to

$$
J=-A^{*} T^{2} \exp \left(-q \phi_{\mathrm{E}}\left(V_{\mathrm{app}}\right) / k T\right),
$$

which can be rewritten in the form

$$
\ln \left(-\frac{I}{T^{2}}\right)=\ln \left(A A^{*}\right)-\frac{q \phi_{\mathrm{E}}\left(V_{\mathrm{app}}\right)}{k T},
$$

for barrier height analysis from Arrhenius plots, $I$ is the current through the device and $A$ is the effective contact area.

Here it is assumed that the quasi-Fermi level for electrons remains horizontal throughout the depletion region. This assumption is valid if the electron mean free path exceeds the distance within which the barrier falls by $k T / q$ from its maximum value $^{1}$. This is likely the case for our study as we have high-mobility materials and from previous studies the mean free path is known to exceed $50 \mathrm{~nm}$ for similar semiconductor NW systems ${ }^{49}$. In addition, the barrier height is assumed to be temperature-independent ${ }^{19}$. This is likely the case for our study as we obtain straight lines in the Arrhenius plots, see Fig. $3 \mathrm{~b}$.

Band diagram reconstruction for series A NWs. The band diagram reconstruction was done by solving the one-dimensional Poisson equation at zero bias and $300 \mathrm{~K}$ (ref. 20). We used bulk material parameters, since the Fermi wavelength of electrons in the NWs is much smaller than the NW diameter ${ }^{49}$. In accordance with TEM studies, the GaAs segment length was taken to be $25 \mathrm{~nm}$ and the InGaAs transition region to be $80 \mathrm{~nm}$. InAs was considered to be $n$-type with a doping concentration of $10^{17} \mathrm{~cm}^{-3}$, and GaAs was considered to be $p$-type with the same doping concentration. The doping concentration was interpolated for the graded InGaAs segment. Indeed, it was previously demonstrated that epitaxial InAs NWs are unintentionally degenerately $n$-type doped $\left(10^{17} \mathrm{~cm}^{-3}\right)^{29}$, whereas GaAs NWs become $p$-type. Residual carbon was suggested to be the source of doping in both InAs and GaAs NWs, in agreement with the amphoteric behaviour of carbon ${ }^{30}$.

Barrier height analysis for series B NWs. Series B NWs with different doping profiles (fully $n$-type, half-way-doped $n$-type and nominally undoped) were characterized with $I-V$ measurements using $\mathrm{STM}^{33}$. Several NWs of the same type were characterized in this way.

For STM characterization, the exact position of as-grown GaAs NWs standing vertically on the substrate was first determined with the STM ${ }^{50}$. Subsequently, the STM tip was gently pressed directly into the catalytic Au particle of an individual GaAs NW, with a precaution not to damage the $\mathrm{NW}^{51}$. By this procedure an ohmic point contact between the metallic STM tip (etched tungsten wire sputtered in vacuum) and the catalytic particle was established ${ }^{51}$. The GaAs substrate was used as the other contact for the $I-V$ measurements. The STM system allowed electrical characterization only at room temperature $(300 \mathrm{~K})$ in darkness.

The accuracy of the Schottky barrier height extraction with this method is limited by the uncertainty of Richardson's constant ${ }^{19}$. For our analysis, $A^{*}=7 \pm 3 \mathrm{~A} \mathrm{~cm}^{-2} \mathrm{~K}^{-2}$ was extracted from Arrhenius plots of the $\mathbf{A}$ series samples for $V_{\text {app }}>0$. This value is comparable to the values theoretically predicted $^{52}$ and experimentally observed for $\mathrm{GaAs}^{53,54}$.

We obtained $q \phi_{\mathrm{S}}=0.32 \pm 0.04 \mathrm{eV}$ for nominally undoped NWs from series $\mathbf{B}$. For fully $n$-type NWs the electrical current was a few orders of magnitude higher than that for half-way-doped $n$-type NWs and there was almost no rectification indicating that the interface between free-standing GaAs NWs and GaAs substrate did not affect our Schottky barrier height measurements for half-way-doped $n$-type NWs. Furthermore, the same Schottky barrier height was obtained for half-waydoped $n$-type and nominally undoped NWs indicating that the interface between free-standing GaAs NWs and GaAs substrate also did not affect our Schottky barrier height measurements for nominally undoped NWs.

Using the same analysis method of extracting the barrier height at $300 \mathrm{~K}$ on series A NWs gives a barrier height of $0.56 \pm 0.03 \mathrm{eV}$, which is consistent with the zero bias barrier in Fig. 3c.
Electric dipole layer influence on the Schottky barrier. The electric dipole layer is formed because of the termination of the GaAs NW by As atoms at the contact interface with the Au catalyst. In the following, we estimate the potential energy difference caused by the electric dipole layer, $q d \phi$. According to the Pauling scale, the electronegativity of $\mathrm{Ga}$ and $\mathrm{As}$ is 1.77 and 2.25 , respectively ${ }^{55}$, and the ionicity of the $\mathrm{Ga}-\mathrm{As}$ chemical bond in GaAs amounts to $\omega=0.31$ (ref. 56). The electronegativity difference leads to an electric dipole layer formation at the Aucatalyst/GaAs-NW interface with a step in the electric potential. Let us consider the electric dipole layer as a parallel plate capacitor with a corresponding capacitance $C=\frac{\varepsilon_{0} A}{l}$, where $\epsilon_{0}=8.85 \times 10^{-12} \mathrm{~F} \mathrm{~m}^{-1}$ is the dielectric constant, $A$ is the effective contact area between the catalytic particle and the NW and $l$ is the thickness of the electric dipole layer. Using the definition of electric capacitance $C=Q / d \phi$, where $Q$ is the charge at a potential difference $d \phi$, we can rewrite the capacitor equation as

$$
d \phi=\frac{Q l}{\varepsilon_{0} A}
$$

The electric dipole layer can be characterized by its dipole moment $\boldsymbol{\mu}=q_{\mathrm{e}} \mathbf{l}$, where $q_{\mathrm{e}}$ is the effective electric charge and $|\mathbf{1}|=l$ can be calculated from the crysta structure of GaAs as $l=\frac{a \sqrt{3}}{4}=2.45 \AA$, where $a=5.65 \AA$ is the lattice constant of $\mathrm{GaAs}^{19}$. Taking into account the chemical bond ionicity, equation (6) can be written in the following form:

$$
d \phi=\frac{N_{\mathrm{S}} q_{\mathrm{e}} l \omega}{\varepsilon_{0}},
$$

where $N_{\mathrm{s}}=8.85 \times 10^{18} \mathrm{~m}^{-2}$ is the atomic surface density as calculated from the crystal structure of GaAs and $q_{\mathrm{e}}=0.2 \cdot q=3.2 \times 10^{-20} \mathrm{C}$ is the effective electric charge for one Ga-As ionic bond ${ }^{57}$. Equation (7) yields the potential difference $d \phi=2.43 \mathrm{~V}$, which corresponds to a potential energy difference $q d \phi=2.43 \mathrm{eV}$.

The ideal Schottky model (Fig. 5a) predicts a Schottky barrier for the In-alloyed Au-catalyst $/ \mathrm{GaAs}-\mathrm{NW}$ interface of $q \phi_{\mathrm{S}(\mathrm{AuIn})}=q \phi_{\mathrm{AuIn}}-q \chi_{\mathrm{GaAs}}=0.89 \mathrm{eV}$ $\left(q \phi_{\text {AuIn }}=4.96 \mathrm{eV}\right.$ is the work function for an AuIn alloy with an In content of 30 at.\% (ref. 39), $q \chi_{\mathrm{GaAs}}=4.07 \mathrm{eV}$ (ref. 38) is the affinity for GaAs).

The electric field associated with the formed electric dipole layer leads to a step in the potential energy at the interface (As atoms in contact with Au-catalyst have higher electronegativity than Ga atoms). In the ideal Schottky model, this step shifts the conduction band edge of GaAs below the Fermi level in the Au-catalyst particle (Fig. 5b) by an amount $q \Delta=0.5 \cdot q d \phi-q \phi_{\mathrm{S}(\text { AuIn) }}=0.33 \mathrm{eV}$.

From our experiments we extract a Schottky barrier height between the $\mathrm{Au}$ catalyst and GaAs NW of $q \phi_{\mathrm{S}}=0.35 \mathrm{eV}$. We now assume that pinning states at the interface account for the potential energy $q d \phi_{\text {States }}=q \phi_{\mathrm{S}}+q \Delta=0.68 \mathrm{eV}$. From this assumption, the density of pinning states can readily be estimated if we again consider an electric dipole layer, now formed by the surface states and the charges induced by them in the metal particle ${ }^{5}$. If we substitute $Q / A=n \cdot q$ in equation (6), where $n$ is the density of surface states, then $n$ can be assessed as:

$$
n=\frac{\varepsilon_{0} q d \phi_{\text {States }}}{q l}
$$

Equation (8) yields a surface density of states $\sim 10^{17} \mathrm{~m}^{-2}$ at the Au-catalyst/ GaAs interface.

\section{References}

1. Rhoderick, E. H. Metal-semiconductor contacts. Solid State and Electron Devices IEE Proc. I 129, 1-14 (1982).

2. Baca, A., Ren, F., Zolper, J., Briggs, R. \& Pearton, S. A survey of ohmic contacts to III-V compound semiconductors. Thin Solid Films 308, 599-606 (1997).

3. Tung, R. T. Recent advances in Schottky barrier concepts. Mater. Sci. Eng. $R$ Rep. 35, 1-138 (2001).

4. Landman, U., Barnett, R. N., Scherbakov, A. G. \& Avouris, P. Metalsemiconductor nanocontacts: silicon nanowires. Phys. Rev. Lett. 85, 1958-1961 (2000).

5. Léonard, F. \& Tersoff, J. Role of Fermi-level pinning in nanotube Schottky diodes. Phys. Rev. Lett. 84, 4693-4696 (2000).

6. Malagù, C., Guidi, V., Carotta, M. C. \& Martinelli, G. Unpinning of Fermi level in nanocrystalline semiconductors. Appl. Phys. Lett. 84, 4158-4160 (2004).

7. Léonard, F. \& Talin, A. A. Size-dependent effects on electrical contacts to nanotubes and nanowires. Phys. Rev. Lett. 97, 026804 (2006).

8. Léonard, F. \& Talin, A. A. Electrical contacts to one- and two-dimensional nanomaterials. Nat. Nanotech. 6, 773-783 (2011).

9. Coss, B. E. et al. Measurement of Schottky barrier height tuning using dielectric dipole insertion method at metal-semiconductor interfaces by photoelectron spectroscopy and electrical characterization techniques. J. Vac. Sci. Technol. B 31, 021202 (2013).

10. Blank, T. \& Gol'Dberg, Y. A. Mechanisms of current flow in metalsemiconductor ohmic contacts. Semiconductors 41, 1263-1292 (2007)

11. Léonard, F., Talin, A. A., Swartzentruber, B. S. \& Picraux, S. T. Diameterdependent electronic transport properties of Au-catalyst/Ge-nanowire Schottky diodes. Phys. Rev. Lett. 102, 106805 (2009).

12. Sheldon, M. T., Trudeau, P. E., Mokari, T., Wang, L. W. \& Alivisatos, A. P. Enhanced semiconductor nanocrystal conductance via solution grown contacts. Nano Lett. 9, 3676-3682 (2009). 
13. Han, N. et al. GaAs nanowire Schottky barrier photovoltaics utilizing Au-Ga alloy catalytic tips. Appl. Phys. Lett. 101, 013105 (2012).

14. Howes, M. J. \& Morgan, D. V. Gallium Arsenide: Materials, Devices, and Circuits (Wiley-Interscience, 1985).

15. Persson, A. I. et al. Solid-phase diffusion mechanism for GaAs nanowire growth. Nat. Mater. 3, 677-682 (2004).

16. Wallentin, J. \& Borgström, M. T. Doping of semiconductor nanowires. J. Mater. Res. 26, 2142-2156 (2011).

17. Suyatin, D. B., Thelander, C., Björk, M. T., Maximov, I. \& Samuelson, L. Sulfur passivation for ohmic contact formation to InAs nanowires. Nanotechnol. 18, 105307 (2007).

18. Blank, T., Gol'dberg, Y. A., Konstantinov, O., Nikitin, V. \& Posse, E. Mechanism of current flow in alloyed ohmic In/GaAs contacts. Tech. Phys. 52, 285-287 (2007).

19. Schroder, D. K. Semiconductor Material and Device Characterization 3rd edn (Wiley-Interscience, 2006).

20. Tan, I. H., Snider, G. L., Chang, L. D. \& Hu, E. L. A selfconsistent solution of Schrödinger-Poisson equations using a nonuniform mesh. J. Appl. Phys. 68, 4071-4076 (1990).

21. Waldrop, J. Schottky-barrier height of ideal metal contacts to GaAs. Appl. Phys. Lett. 44, 1002-1004 (1984).

22. Spitzer, W. G. \& Mead, C. A. Barrier height studies on metal-semiconductor systems. J. Appl. Phys. 34, 3061-3069 (1963).

23. Sato, T., Kasai, S., Okada, H. \& Hasegawa, H. Electrical properties of nanometer-sized Schottky contacts on $\mathrm{n}-\mathrm{GaAs}$ and $\mathrm{n}$-InP formed by in situ electrochemical process. Jpn J. Appl. Phys. 39, 4609-4615 (2000).

24. Suyatin, D. B. et al. Electrical properties of self-assembled branched InAs nanowire junctions. Nano Lett. 8, 1100-1104 (2008).

25. Sullivan, J., Tung, R., Pinto, M. \& Graham, W. Electron transport of inhomogeneous Schottky barriers: a numerical study. J. Appl. Phys. 70, 7403-7424 (1991).

26. Jabeen, F. et al. Contactless monitoring of the diameter-dependent conductivity of GaAs nanowires. Nano Res. 3, 706-713 (2010).

27. Han, N. et al. Controllable p-n switching behaviors of GaAs nanowires via an interface effect. ACS Nano 6, 4428-4433 (2012).

28. Wieder, H. Problems and prospects of compound semiconductor field-effect transistors. J. Vac. Sci. Technol. 17, 1009-1018 (1980).

29. Thelander, C. et al. Single-electron transistors in heterostructure nanowires. Appl. Phys. Lett. 83, 2052-2054 (2003).

30. Thelander, C. et al. Electron transport in InAs nanowires and heterostructure nanowire devices. Solid State Commun. 131, 573-579 (2004).

31. Hudait, M., Venkateswarlu, P. \& Krupanidhi, S. Electrical transport characteristics of $\mathrm{Au} / \mathrm{n}-\mathrm{GaAs}$ Schottky diodes on $\mathrm{n}-\mathrm{Ge}$ at low temperatures. Solid State Electron. 45, 133-141 (2001).

32. Sze, S. Semiconductor Devices-Physics and Technology (John Wiley \& Sons, 1985).

33. Talin, A. A. et al. Transport characterization in nanowires using an electrical nanoprobe. Semicond. Sci. Technol. 25, 024015 (2010).

34. Cowley, A. \& Sze, S. Surface states and barrier height of metal-semiconductor systems. J. Appl. Phys. 36, 3212-3220 (1965).

35. Sze, S. M. \& Ng, K. K. Physics of Semiconductor Devices 3rd edn (John Wiley \& Sons, 2006).

36. Michaelson, H. B. The work function of the elements and its periodicity. J. Appl. Phys. 48, 4729-4733 (1977).

37. Lodha, S., Janes, D. B. \& Chen, N.-P. Fermi level unpinning in ex situ Schottky contacts on n-GaAs capped with low-temperature-grown GaAs. Appl. Phys. Lett. 80, 4452-4454 (2002).

38. Levinshtein, M. E. \& Rumyantsev, S. L. Handbook Series on Semiconductor Parameters Vol. 1 (World Scientific, 1996).

39. Rothschild, J. A. \& Eizenberg, M. Work function calculation of solid solution alloys using the image force model. Phys. Rev. B 81, 224201 (2010).

40. Bardeen, J. Surface states and rectification at a metal semi-conductor contact. Phys. Rev. 71, 717-727 (1947).

41. Gurwitz, R. et al. Bandgap and band discontinuity in wurtzite/zincblende GaAs homomaterial heterostructure. Appl. Phys. Lett. 100, 191602 (2012).

42. Hjort, M. et al. Direct imaging of atomic scale structure and electronic properties of GaAs wurtzite and zinc blende nanowire surfaces. Nano Lett. 13, 4492-4498 (2013).

43. Björk, M. et al. One-dimensional heterostructures in semiconductor nanowhiskers. Appl. Phys. Lett. 80, 1058-1060 (2002).
44. Kahng, D. Au-n-Type GaAs Schottky barrier and its varactor application. Bell Syst. Tech. J. 215-224 (1964).

45. Tung, R. T. Chemical bonding and Fermi level pinning at metal-semiconductor interfaces. Phys. Rev. Lett. 84, 6078-6081 (2000).

46. Zhang, Z. \& Yates, Jr J. T. Band bending in semiconductors: chemical and physical consequences at surfaces and interfaces. Chem. Rev. 112, 5520-5551 (2012).

47. Storm, K. et al. Spatially resolved Hall effect measurement in a single semiconductor nanowire. Nat. Nanotech. 7, 718-722 (2012).

48. Magnusson, M. H., Deppert, K., Malm, J.-O., Bovin, J.-O. \& Samuelson, L. Gold nanoparticles: production, reshaping, and thermal charging. J. Nanopart. Res. 1, 243-251 (1999).

49. Hansen, A. E., Björk, M. T., Fasth, C., Thelander, C. \& Samuelson, L. Spin relaxation in InAs nanowires studied by tunable weak antilocalization. Phys. Rev. B 71, 205328 (2005).

50. Fian, A. et al. New flexible toolbox for nanomechanical measurements with extreme precision and at very high frequencies. Nano Lett. 10, 3893-3898 (2010).

51. Larsson, M. W., Wallenberg, L. R., Persson, A. I. \& Samuelson, L. Probing of individual semiconductor nanowhiskers by TEM-STM. Microsc. Microanal. 10, 41-46 (2004).

52. Crowell, C. \& Sze, S. Current transport in metal-semiconductor barriers. Solid State Electron. 9, 1035-1048 (1966).

53. Missous, M., Rhoderick, E. H., Woolf, D. A. \& Wilkes, S. P. On the Richardson constant of intimate metal-GaAs Schottky barriers. Semicond. Sci. Technol. 7, 218-221 (1992).

54. Pilkington, S. J., Missous, M. \& Woolf, D. A. On the Richardson constant of intimate metal-GaAs (111)B Schottky diodes grown by molecular beam epitaxy J. Appl. Phys. 74, 6256-6260 (1993).

55. Bergmann, D. \& Hinze, J. Electronegativity and molecular properties. Angew. Chem. Int. Ed Engl. 35, 150-163 (1996).

56. Phillips, J. C. Ionicity of the chemical bond in crystals. Rev. Mod. Phys. 42, 317-356 (1970).

57. Phillips, J. C. Bonds and Bands in Semiconductors (Academic Press New York, 1973).

\section{Acknowledgements}

We acknowledge financial support from the Nanometer Structure Consortium at Lund University (nmC@LU), the Swedish Research Council (VR), The Knut and Alice Wallenberg Foundation, the European Research Council, the Swedish National Board for Industrial and Technological Development and the Swedish Foundation for Strategic Research. We also acknowledge Dan Csontos for helpful suggestions and fruitful discussions, Jalil Shah for help with STM measurements, Daniel Wallin for input on EBL alignment process, Magnus Borgström and Ann I. Persson for important input on NW growth and doping.

\section{Author contributions}

L.S. conceived and initiated the project. H.P., I.M., R.T., A.M. and L.S. conceived and supervised the experiments. D.B.S. grew the NWs. M.E.M. and J.B.W. performed TEM and XEDS studies. D.B.S. fabricated the series A NW samples and performed electrical measurements and data analysis for the samples. J.T. and D.B.S. performed photocurrent measurements and carried out associated data analysis. O.P., R.T. and A.M. performed the STM-based electrical measurements for series B NWs. V.J. carried out the data analysis for series B NWs. V.A.N., D.B.S. and H.P. elucidated the physics behind the experimental observations. D.B.S., V.J., V.A.N., R.T. and H.P. wrote the manuscript. All authors discussed the results and implications, and commented on the work.

\section{Additional information}

Supplementary Information accompanies this paper at http://www.nature.com/ naturecommunications

Competing financial interests: The authors declare no competing financial interests.

Reprints and permission information is available online at http://npg.nature.com/ reprintsandpermissions/

How to cite this article: Suyatin, D. B. et al. Strong Schottky barrier reduction at Aucatalyst/GaAs-nanowire interfaces by electric dipole formation and Fermi-level unpinning. Nat. Commun. 5:3221 doi: 10.1038/ncomms4221 (2014). 